\title{
Synthesis and Structure Elucidation of the Human tRNA Nucleoside Mannosyl-Queuosine
}

Markus Hillmeier ${ }^{\mathrm{a}}$, Mirko Wagner ${ }^{\mathrm{a}}$, Timm Ensfelder ${ }^{\mathrm{a}}$, Eva Korytiakova ${ }^{\mathrm{a}}$, Peter Thumbs ${ }^{\mathrm{a}}$, Markus Müller, and Thomas Carell ${ }^{\mathrm{a}, \#}$

a) Department Chemie, Ludwig-Maximilians-Universität München, Butenandtstraße 5-13, 81377 München

Correspondence should be addressed to:

Thomas Carell

E-Mail: thomas.carell@Imu.de

Telefone: $+49(0) 89218077755$

Fax: $+49(0) 89218077756$

Webpage: www.carellgroup.de

\begin{abstract}
Queuosine $(Q)$ is a structurally complex, non-canonical RNA nucleoside. It is present in many eukaryotic and bacterial species, where it is part of the anticodon loop of certain tRNAs. In higher vertebrates, including humans, two further modified queuosine-derivatives exist - galactosyl- (galQ) and mannosylqueuosine (manQ). The function of these low abundant hypermodified RNA nucleosides remains unknown. While the structure of galQ was elucidated and confirmed by total synthesis, the reported structure of manQ still awaits confirmation. By combining total synthesis and LC-MS-co-injection, together with a metabolic feeding study of labelled hexoses, we show here that the natural compound manQ isolated from mouse liver deviates from the literature-reported structure. The chemical structure of the natural product manQ features a novel $\alpha$-allyl connectivity. The data reported here shows that the yet unidentified glycosylases that attach galactose and mannose to the Q-base have different constitutional connectivity preferences. Knowing the correct structure of manQ will now pave the way towards further elucidation of its biological function.
\end{abstract}




\section{Introduction}

Ribonucleic acid (RNA) is a central molecule of life, linking the genotype to the phenotype by integrating both catalytic and coding properties in the synthesis of proteins. ${ }^{1,2}$ To fulfil the plethora of functions known for RNA today, a huge chemical diversity has developed regarding the nucleobases through the evolution of life. ${ }^{3,4}$ The most densely modified RNA molecules are the tRNAs. ${ }^{5}$ Those small adapter molecules feed the required amino acids to the growing peptide chain in the ribosome. Of particular interest are the non-canonical nucleosides that are found in the anticodon loop of tRNAs, in particular at position 34 , known as the Wobble position of the anticodon. ${ }^{6}$ Here, any chemical modification has a direct impact on the coding potential of the tRNA. ${ }^{6-9}$ Queuosine $(\mathbf{1}, Q$, Fig. $\mathbf{1 a})$ is a particularly complex non-canonical nucleoside. ${ }^{10-12}$ It is found in many bacterial and eukaryotic species, where it is located at position 34 of the anticodon loop of tRNA ${ }^{\text {Tyr }}$, $\operatorname{tRNA}^{\text {Asp }}$, tRNA ${ }^{\text {His }}$, and tRNA ${ }^{\text {Asn }} .{ }^{13,14}$
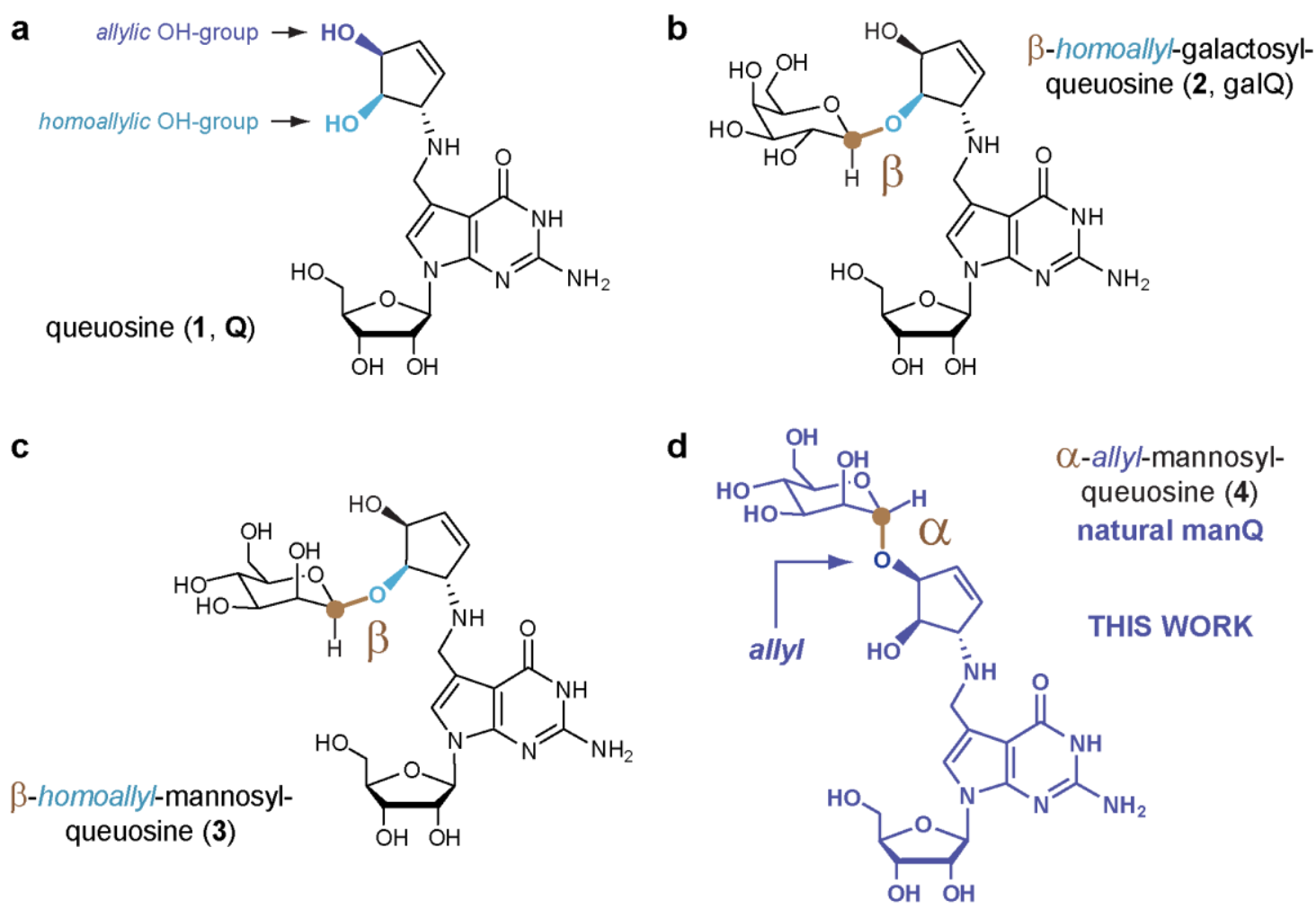

Figure 1. Nucleosides of the queuosine family present in higher vertebrates. a, Queuosine 1 is derived from a 7-deazaguanosine base with a $1(S)$-amino-2(R),3(S)-dihydroxycyclopent-4-ene unit attached via a methylene linker to C7. Possible glycosylation sites are depicted in dark blue (allylic hydroxyl group) and light blue (homoallylic hydroxyl group); b, Structure of the naturally occurring nucleoside $\beta$-homoallyl-galQ 2; c, Structure $\mathbf{3}$ shows the originally proposed structure of manQ, which we revise in this work; $\mathbf{d}$, Corrected structure of natural manQ $\mathbf{4}$ featuring an $\alpha$-allylic connectivity. 
In eukaryotes, queuosine $\mathbf{1}$ is found in both cytosolic and mitochondrial tRNA. ${ }^{15}$ It is biochemically derived from guanosine $(G)$ and characterized by the exchange of the N7-nitrogen for a C7-carbon atom and the addition of a $1(S)$-amino-2(R),3(S)-dihydroxycyclopent-4-ene unit to the C7-position via a methylene linker. ${ }^{12,16}$ The presence of the Q-base in position 34 of the anticodon equips the corresponding tRNAs with the possibility to decode synonymous codons by wobble base pairing. ${ }^{17,18}$ The Q-base is furthermore affecting translational speed, ${ }^{19}$ decoding fidelity, ${ }^{20}$ and it is essential for survival particularly in the absence of sufficient tyrosine. ${ }^{21,22}$ Eukaryotes are unable to biosynthesize $Q$, which forces them to acquire it from prokaryotic sources. ${ }^{21,23-25}$ This establishes a link between the central process of translation and the gut microbiome. ${ }^{26}$ Defective RNA modification processes are increasingly recognized as drivers for severe diseases. ${ }^{27,28}$

In higher vertebrates, including humans, two glycosylated queuosine derivatives exist, which have yet unidentified functions. The first of these hypermodified $Q$ derivatives is found in cytosolic tRNA ${ }^{\text {Tyr }}$, where the Q-base is modified with a galactose residue (galQ, 2, Fig. 1b). ${ }^{29,10}$ The cytosolic tRNA ${ }^{\text {Asp }}$, in contrast, contains a Q-derivative, which is suggested to be mannosylated (manQ, Fig. 1c, d). ${ }^{29}$ Here, we report the total synthesis of the "human natural product" manQ and show that the originally proposed structure 3 (Fig. 1c) needs to be revised in two important aspects: In contrast to the original structure suggestion, we show that the mannose glycosidic bond is not $\beta$ - but $\alpha$-configured. Furthermore, and in contrast to $\beta$-galQ, the mannose is attached not to the homoallylic, but to the allylic hydroxyl group at C3. manQ has consequently the structure $\mathbf{4}$ shown in Fig. $1 \mathrm{~d}$. This result reveals that the yet unknown galactosyl and mannosyl transferases that attach the respective hexose to the Q-nucleobase in tRNA ${ }^{\text {Tyr }}$ and tRNA ${ }^{\text {Asp }}$, are able to differentiate the two hydroxyl groups (allyl vs. homoallyl) at the cyclopentene substructure for yet unknown reasons.

\section{Main Text:}

Synthesis of literature-reported manQ. Because the molecules galQ and manQ are found only in the anticodon loop of one cytosolic tRNA each, ${ }^{30}$ the amounts that can be isolated from nature (e.g. porcine liver) are so small that a structure elucidation based solely on isolated material is practically impossible. Therefore, synthesis of the proposed manQ compound and comparison of the synthetic standard with natural material by LC-MS was the method of choice for us to investigate the manQ structure. The synthesis of compound $\mathbf{3}$, proposed to be natural manQ, was performed as depicted in Fig. 2a. 
a
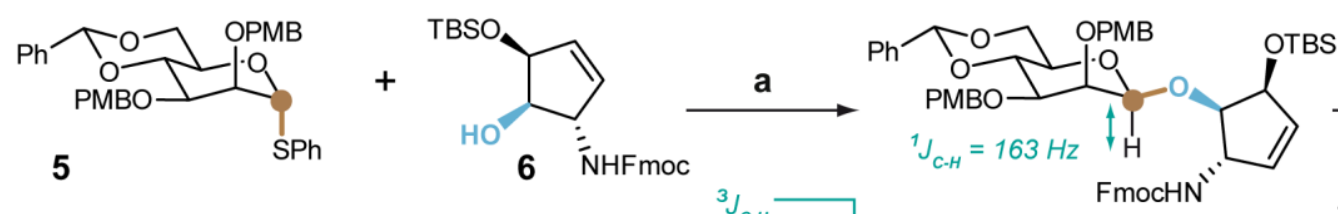

b, $c, d$

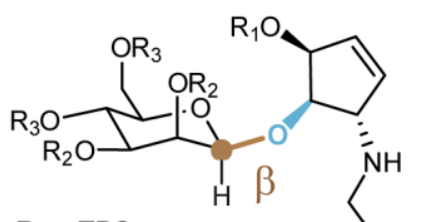

$6 \overline{\mathrm{NHFmoc}}$

FmocHN"

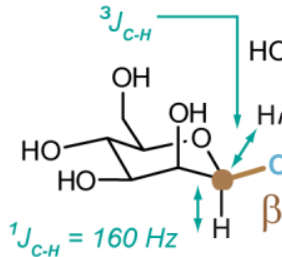

7

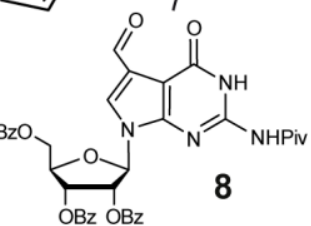

$R_{1}=T B S$

$R_{2}=P M B$

$\mathrm{R}_{3}=\mathrm{CHPh}$

$\mathrm{R}_{4}=$ Piv

$\mathrm{R}_{5}=\mathrm{Bz}$

$\stackrel{\mathrm{N}}{\mathrm{NH}}^{\mathrm{N}}$

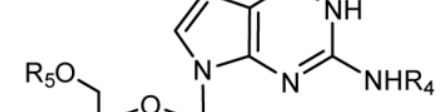

9

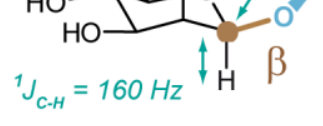

\section{$e, f, g$}

HO b

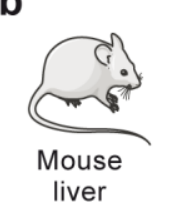

Isolation of total RNA

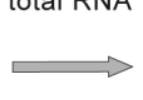

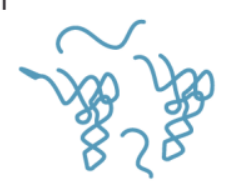

mRNA

tRNA

rRNA

etc.

Enzymatic

digestion

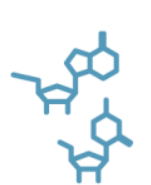

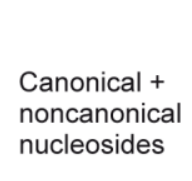

Spiking

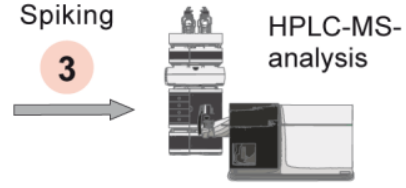

C

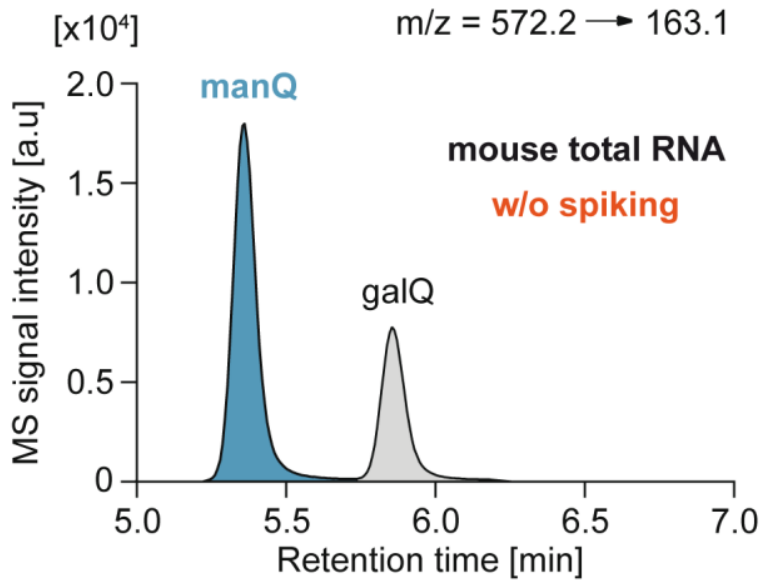

[x10 $]$

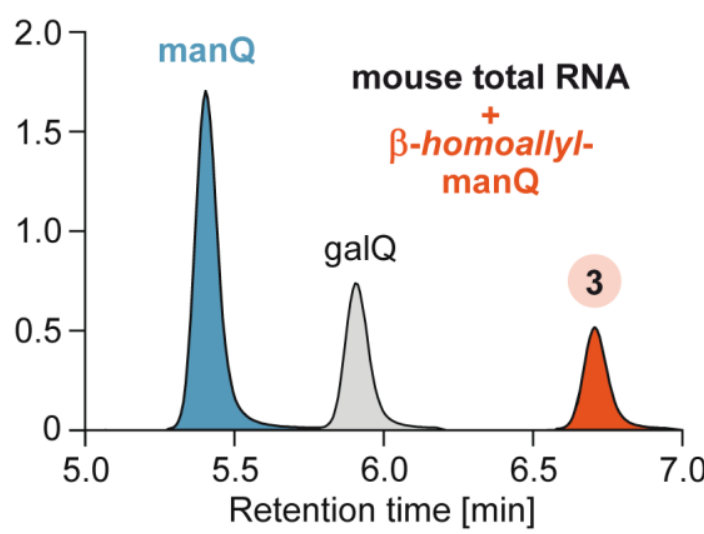

2.0

Figure 2. Attempt to confirm the originally proposed structure of manQ. a, Synthetic route towards $\beta$-homoally/man Q featuring a $\beta$-selective mannosylation key step; a: AgOTf, PhSCl, DTBMP, DCM, $-78^{\circ} \mathrm{C}, 2 \mathrm{~h}, 62 \%$; b: $\mathrm{HNMe}_{2}$, THF, rt, 1 h; c: 8, MeOH, rt, 5 h; d: $\mathrm{NaBH}_{4}, \mathrm{MeOH}, 0{ }^{\circ} \mathrm{C}, 1$ h; e: NaOMe, MeOH, rt, 3 h; f: TFA, DCM, $0{ }^{\circ} \mathrm{C}, 20$ min; $\mathrm{g}$ : HF - pyridine, pyridine, rt, $18 \mathrm{~h}, 22 \%$ (5 steps); $\mathbf{b}$, Workflow for the comparison of the synthetic $\beta$-homoallylmanQ standard with natural manQ via isolation of total RNA from mouse liver, digestion to the nucleoside level, and LC-MS-co-injection experiments; $c$, Chromatograms resulting from the co-injection experiments analysed by UHPLC-MS/MS; Left: Digested total RNA from mouse liver shows two peaks, natural manQ and galQ; Right: Digested total RNA from mouse liver spiked with the synthetic standard $\beta$-homoallyl-manQ $\mathbf{3}$ shows the appearance of an additional peak, thereby disproving the originally proposed structure for manQ. 
For the synthesis of the difficult-to-access $\beta$-mannosyl connectivity, we employed the Crich-method ${ }^{31}$, which required the preparation of the mannosyl donor $\mathbf{5}$. This was achieved along the literaturereported synthesis. ${ }^{31,32}$ The mannosylation reaction of the Fmoc- and TBS- protected 1(S)-amino2(R),3(S)-dihydroxycyclopent-4-ene unit 6 with the mannosyl donor 5 required an activation with $\mathrm{Ag}$ triflate in the presence of phenylthiochloride and 2,6-di-tert-butyl-4-methylpyridine (DTBMP) at $-78^{\circ} \mathrm{C}$ in dry dichloromethane. The reaction gave the mannoside 7 in $62 \%$ yield, and the $\beta$-configuration of its anomeric centre was confirmed by NMR-spectroscopy based on the coupling constant of ${ }^{1} \mathrm{~J}_{\mathrm{C} 1-\mathrm{H} 1}=163 \mathrm{~Hz}$, which is typical for $\beta$-mannosides. ${ }^{33}$ We next cleaved the Fmoc group of the mannoside 7 with dimethylamine (10\% DMA in THF, rt, $1 \mathrm{~h}$ ) and performed the reductive amination with the benzoyl- and pivaloyl-protected 7-formyl-7-deazaguanosine compound 8 ( $\mathrm{MeOH}, \mathrm{rt}, 5 \mathrm{~h}$, then $\mathrm{NaBH}_{4}$, $0{ }^{\circ} \mathrm{C}, 1 \mathrm{~h}$ ) that was prepared as recently described by us. ${ }^{10}$ This afforded the fully protected manQ compound 9. Cleavage of the benzoyl-protecting groups at the ribose was performed under Zemplénconditions ( $0.5 \mathrm{M} \mathrm{NaOMe}, \mathrm{MeOH}, \mathrm{rt}, 5 \mathrm{~h}$ ). The PMB-ethers were deprotected with trifluoro acetic acid in dichloromethane $\left(10 \%, 0{ }^{\circ} \mathrm{C}, 20 \mathrm{~min}\right)$. Finally, we removed the TBS-protecting groups with $\mathrm{HF}$ in pyridine ( $\mathrm{rt}, 18 \mathrm{~h}$ ). This furnished the final manQ compound 3 with mannose being $\beta$-configured and attached to the homoallylic hydroxyl group. As expected, compound $\mathbf{3}$ has a ${ }^{1} \mathrm{~J}_{\mathrm{C}-\mathrm{H}}$-coupling constant of $160 \mathrm{~Hz}$ at the anomeric centre, thereby proving its $\beta$-configuration. Furthermore, the observed ${ }^{3} \mathrm{~J}_{\mathrm{C}-\mathrm{H}^{-}}$ $\mathrm{HMBC}$ coupling in compound $\mathbf{3}$ between the mannosyl $\mathrm{C} 1$-carbon and the homoallylic hydrogen $\mathrm{C} 4 \mathrm{H}$ of the cyclopentene ring (see Supplementary Information 4) unambiguously proved that the mannosyl residue is indeed connected to the homoallylic hydroxyl group.

In order to compare the synthetic manQ compound $\mathbf{3}$ with the natural material, we performed an LCMS co-injection study. To this end, we isolated the total RNA from mouse liver and enzymatically digested this RNA down to the individual nucleosides as recently described by us. ${ }^{10}$ This procedure afforded a mixture of all nucleosides present in the total RNA pool. Subsequent analysis of the resulting nucleoside mixture was performed via UHPLC-MS/MS with a triple quadrupole mass spectrometer (QQQ) set to monitor a specific molecular fragmentation reaction of the glycosylated Q-derivatives. Using collision-induced dissociation, these nucleosides undergo two heterolytic bond dissociations, leading to a loss of both the ribose sugar and the glycosylated cyclopentene unit. Monitoring the corresponding mass transition of $\mathrm{m} / \mathrm{z}=572.2 \rightarrow 163.1$ allows a sensitive detection of these compounds (see Fig. $\mathbf{2 b}$ and Supplementary Figure 1). With an UHPLC separation gradient of $0 \rightarrow 2 \%$ (v/v) $\mathrm{MeCN} / \mathrm{H}_{2} \mathrm{O}$ in $0 \rightarrow 8 \mathrm{~min}$ on a Poroshell $120 \mathrm{SB}-\mathrm{C8}$ column, all hexose-modified queuosine derivatives can be separated chromatographically (see Fig. 2c). Indeed, when analysing the mouse liver sample, we detected two clearly separated signals in the RNA nucleoside pool with galQ 2 eluting at $5.9 \mathrm{~min}$, and the natural manQ compound eluting at $5.4 \mathrm{~min}$, respectively. The compound eluting at 5.9 min was unambiguously identified as galQ $\mathbf{2}$ with the help of a synthetic reference compound (Supporting Fig. 2). Upon co-injection of the synthetic material 3, we were expecting to see again two signals with the manQ signal having gained in intensity. To our surprise, however, we noted that this is not the case. Instead, the co-injection experiment provided a third signal with a retention time of 
$6.8 \mathrm{~min}$, clearly separated from both natural manQ and galQ 2. This result unequivocally shows that manQ 3 is NOT identical to the natural manQ compound. Therefore, the structure proposal for manQ reported in literature must be incorrect.

Puzzled by this result, we initially reasoned that the compound originally identified as manQ may contain a different sugar than mannose, even though a previous study showed that a mannosyl moiety can enzymatically be transferred to the Q-base from GDP-mannose. ${ }^{34}$ To verify that manQ contains indeed a mannose sugar, we performed a metabolic labelling study (Fig. 3a): We added different isotope-labelled sugars to a HEK293T cell culture and subsequently isolated the total RNA of the cells to see if the natural manQ had incorporated the isotope labels. During these experiments, the cell culture medium was additionally supplemented with high concentrations of unlabelled glucose or mannose as carbon source in order to suppress the metabolic conversion of the labelled sugar into other carbohydrates. Without these metabolic suppressors, such conversion would initiate an isotope scrambling process that would jeopardize the experiments. The results of our study are depicted in Figure 3b. It is clearly evident that feeding of ${ }^{13} \mathrm{C}_{6}$-galactose and ${ }^{13} \mathrm{C}_{6}$-glucose in the presence of the metabolic suppressors glucose or mannose, respectively, gave little or no incorporation of ${ }^{13} \mathrm{C}$ into the isolated natural manQ compound. In contrast, feeding of ${ }^{13} \mathrm{C}_{6}$-mannose in combination with the metabolic suppressor glucose quickly led to the time-dependent formation of ${ }^{13} \mathrm{C}_{6}$-man $\mathrm{Q}$, thereby confirming that the sugar connected to the Q-base in manQ is indeed mannose.

a

Unlabelled hexose as suppressor of metabolic conversion

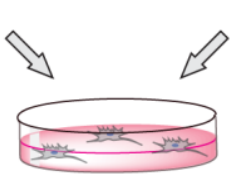

${ }^{13} \mathrm{C}_{6}$-galactose

or

${ }^{13} \mathrm{C}_{6}$-glucose

or

${ }^{13} \mathrm{C}_{6}$-mannose

Isolation of RNA,

Enzymatic digestion, LC-MS analysis

$\mathrm{m} / \mathrm{z}=572.2198 \pm 50$

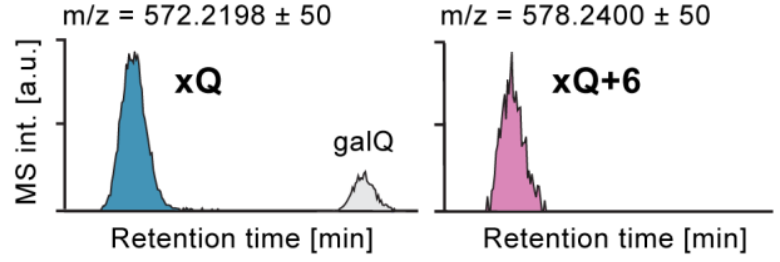

Figure 3. Confirmation of the incorporated hexose sugar as being mannose. a, Workflow of the metabolic labelling study providing ${ }^{13} \mathrm{C}_{6}$-galactose, ${ }^{13} \mathrm{C}_{6}$-glucose, or ${ }^{13} \mathrm{C}_{6}$-mannose in combination with an unlabelled carbon source (glucose or mannose) that suppresses metabolic interconversion of the carbohydrates and hence isotope scrambling; b, Results of the LC-MS experiments showing quick incorporation of heavy sugar when feeding ${ }^{13} \mathrm{C}_{6^{-}}$ mannose in presence of glucose, thereby confirming the identity of $x Q$ as manQ; Error bars represent the standard deviation of three independent experimental replicates

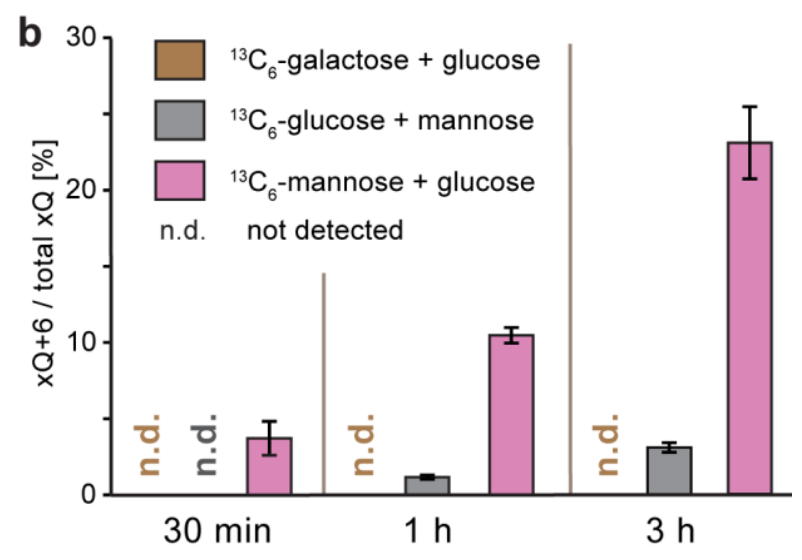


We therefore reasoned that natural manQ differs from the reported structure $\mathbf{3}$ likely regarding the configuration of the anomeric centre and/or the connectivity at the cyclopentene moiety (ally/ versus homoallyl mannoside). With $\mathbf{3}$ proven not to be the natural compound, we were left with the three remaining structures 4 ( $\alpha$-allyl-connectivity), 10 ( $\beta$-allyl-connectivity), and 11 ( $\alpha$-homoallylconnectivity, see Fig. 4a). For their synthesis, it was necessary to first develop syntheses for the properly protected 1-amino-2,3-dihydroxycyclopent-4-enes 13-15 (Fig. 4b). To access these compounds, we started with the Fmoc-protected 1-amino-2,3-dihydroxypent-4-ene 12. For the synthesis of the 1-Fmoc-3-PMB-protected cyclopent-4-ene 13, needed for the synthesis of 11, we first protected in 12 both $\mathrm{OH}$ groups as an anisaldehyde acetal (anisaldehyde dimethylacetal, CSA, rt, $2 \mathrm{~h}$, $92 \%)$, followed by a selective reductive opening of the homoallylic OH group with DIBAL-H. This gave the 1-Fmoc-3-PMB-protected-dihydroxycyclopent-4-ene 13 (DCM, $-78^{\circ} \mathrm{C}, 2 \mathrm{~h}, 85 \%$ ). For 14, we used 13 as the starting material. Protection of the homoallylic hydroxyl group in $\mathbf{1 3}$ with SEM-Cl $\left(\mathrm{NBu}_{4} \mathrm{l}\right.$, pyridine, DMF, $70^{\circ} \mathrm{C}, 18 \mathrm{~h}, 83 \%$ ), followed by cleavage of the $p$-methoxybenzylether (10\% TFA in $\mathrm{DCM}, 0{ }^{\circ} \mathrm{C}, 5 \mathrm{~min}$ ) furnished compound 14 . Reaction of 12 with TBSOTf at $-55^{\circ} \mathrm{C}$ provided after $15 \mathrm{~min}$ predominantly the 1-Fmoc-3-TBS-protected compound 6 as the kinetic product, which we had used before for the synthesis of the literature-reported structure of manQ 3. If this reaction was, however, performed at $-10^{\circ} \mathrm{C}$ for a longer period of time $(2 \mathrm{~h})$, we observed formation of the thermodynamically more stable 1-Fmoc-2-TBS-protected compound 15.

With the differently protected cyclopentenes 13, 14 and 15 in hand, the manQ compounds 10, 11, and 4 were synthesized via the glycosylation and reductive amination approach (Fig. 4c). The first target compound, $\beta$-allyl-manQ 10, was prepared from cyclopentene precursor 13 starting with a stereoselective $\beta$-mannosylation reaction (AgOTf, PhSCl, 2,6-di-tert-butyl-4-methylpyridine, DCM, $78{ }^{\circ} \mathrm{C}, 2 \mathrm{~h}, 52 \%$ ) to give the glycosylated product 17. Fmoc-deprotection (10\% $\mathrm{HNMe}_{2}$ in THF, rt, $1 \mathrm{~h}$ ) and subsequent reductive amination with deazaguanosine precursor 8 gave the fully protected $\beta$-allylmanQ 20. A three-step final deprotection procedure (1: $\mathrm{NaOMe}, \mathrm{MeOH}, 18 \mathrm{~h} ; 2: 10 \%$ TFA in DCM, $0{ }^{\circ} \mathrm{C}, 15 \mathrm{~min} ; 3$ : HF pyridine, MeCN, rt, $18 \mathrm{~h}$ ) gave $\beta$-allyl-manQ 10. For the second target compound, $\alpha$-homoallyl-manQ 11, we started from cyclopentene precursor 14 and employed Schmitt-Sinayglycosylation conditions with the literature-known glycosyl donor 16 (TMSOTf, THF, $0{ }^{\circ} \mathrm{C}$ ) to obtain glycosylated product 18 . The synthesis of $\alpha$-homoallyl-manQ 11 was completed from there by Fmocdeprotection, reductive amination with 8, and a two-step final deprotection (1: $10 \%$ TFA, DCM, 15 min; 2: NaOMe, $\mathrm{MeOH}, \mathrm{rt}, 2$ d). 

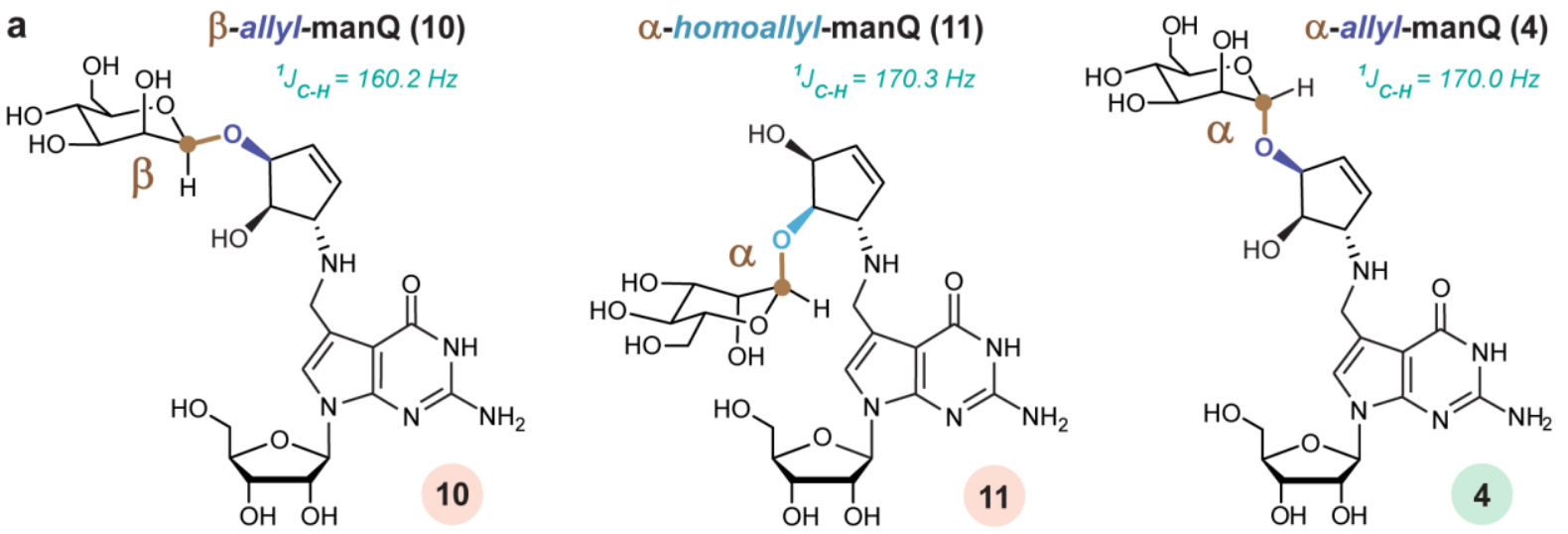

b

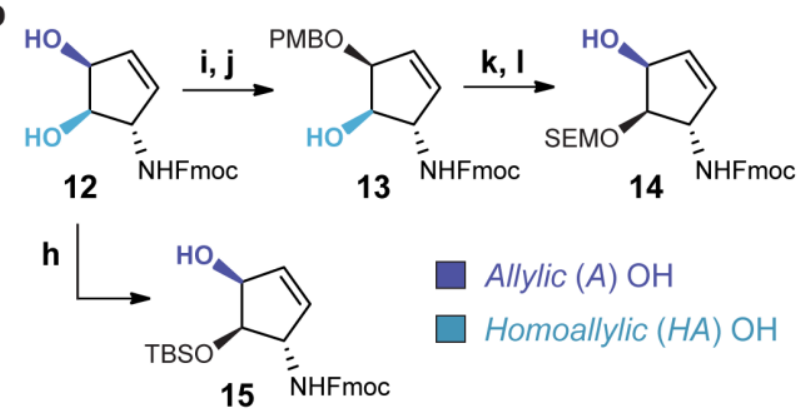

d

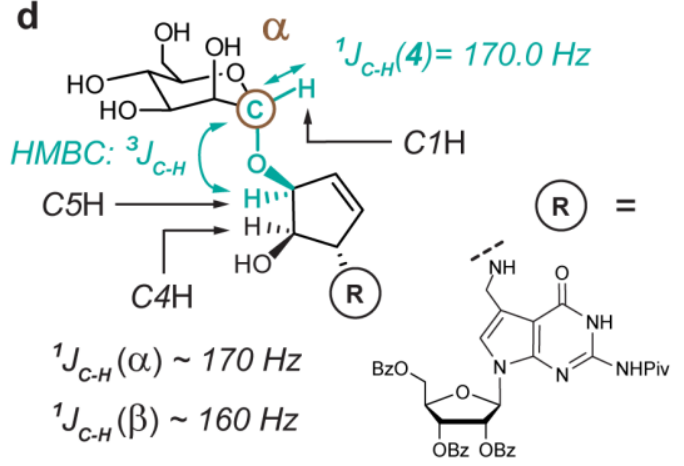

C

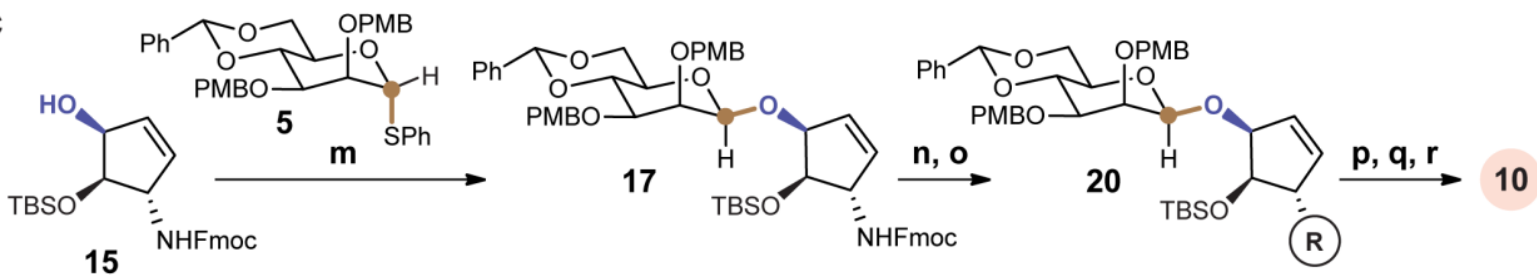

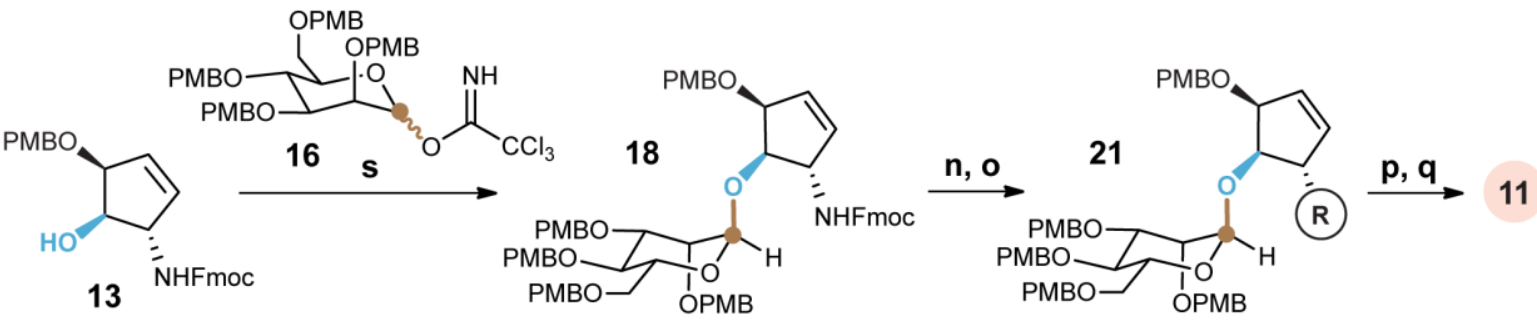

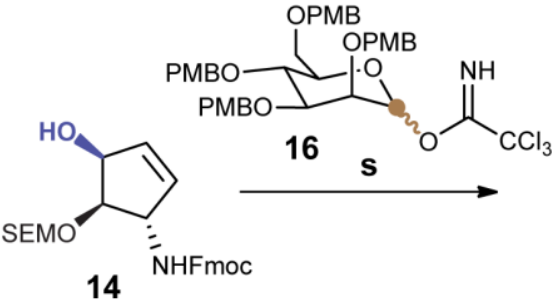

14

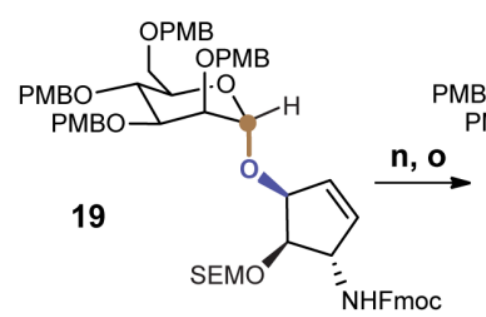

22

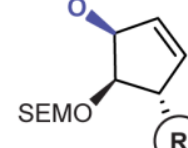<smiles>[13CH3][131Pb]</smiles>

Figure 4. Alternative manQ structures and their syntheses. a, manQ target compounds: $\beta$-allyl-manQ 10, $\alpha$-homoallyl-manQ 11, and the correct structure 4 of natural manQ featuring an $\alpha$-allyl-connectivity; $\mathbf{b}$, Regioselective protection of cyclopentene 12 for the synthesis of precursors 13-15; h: TBSOTf, DMF, $-10{ }^{\circ} \mathrm{C}, 2 \mathrm{~h}$, $41 \%$; i: anisaldehyde dimethyl acetal, CSA, DMF, rt, $2 \mathrm{~h}, 98 \%$; j: DIBAL-H , DCM, $-78{ }^{\circ} \mathrm{C}, 3 \mathrm{~h}, 85 \%$; $\mathrm{k}$ : SEM-Cl, 
$\mathrm{NBu}_{4}$ l, pyridine, DMF, $70{ }^{\circ} \mathrm{C}, 18 \mathrm{~h}, 75 \%$; I: TFA, DCM, $0{ }^{\circ} \mathrm{C}, 5 \mathrm{~min}, 83 \%$; c, Synthesis of the manQ compounds 10 , 11, and 4 from 13, 14 and 15 via an appropriate stereoselective glycosylation step and subsequent reductive amination; m: AgOTf, PhSCl, DTBMP, DCM, $-78^{\circ} \mathrm{C}, 2 \mathrm{~h}, 52 \%$;: DBU, MeCN, rt, $1 \mathrm{~h}$; o: 8, MeOH, 2-5 h, then $\mathrm{NaBH}_{4}, 0^{\circ} \mathrm{C}, 15-30$ min; p: NaOMe, MeOH, rt; q: HF · pyridine, EtOAc, rt, 18 h; r: TFA, DCM, $0{ }^{\circ} \mathrm{C}$; s: TMSOTf, DCM, $0{ }^{\circ} \mathrm{C}$; t: HF - pyridine, MeCN, rt, 1 week; $\mathbf{d}$, Depiction of the ${ }^{1} \mathrm{~J}_{\mathrm{CH}}$-coupling at the anomeric center of $\alpha$-allyl-manQ

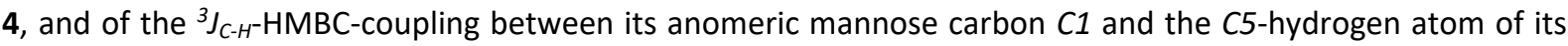
cyclopentene moiety. Both couplings were used to confirm the structure of this compound via NMR, and similar couplings were used to confirm the structures of $\mathbf{3}, \mathbf{1 0}$, and $\mathbf{1 1 .}$

The third target compound, $\alpha$-allyl-manQ 4, was synthesized again via Schmitt-Sinay-glycosylation conditions with donor 16. In this case, the SEM-protected cyclopentene precursor 15 was used, because the TBS-protected precursor 13 proved to be sterically too demanding for the glycosylation reaction. Reaction of $\mathbf{1 5}$ with $\mathbf{1 6}$ however was not fully stereoselective. But after full completion of the synthesis by Fmoc-deprotection, reductive amination, and subsequent three-step deprotection (1: $\mathrm{NaOMe}, \mathrm{MeOH}, \mathrm{rt}, 18 \mathrm{~h} ; 2: 10 \% \mathrm{TFA}, \mathrm{DCM}, 0^{\circ} \mathrm{C}, 15 \mathrm{~min}$; 3: HF $\cdot$ pyridine, MeCN, rt, 1 week), we obtained a mixture of 10 and $\mathbf{4}$, which was separated by reversed-phase HPLC-chromatography (see Supplementary Information 3.4). This allowed us to obtain $\alpha$-allyl-manQ 4 in excellent purity.

We next confirmed the structure of the manQ compounds 4, 10, and 11 using NMR spectroscopy (Fig. 4d). For $\beta$-allyl-manQ 10, we observed a ${ }^{1} J_{C-H}-$ coupling at the mannose anomeric centre of $160.2 \mathrm{~Hz}$, indicative of the $\beta$-configuration. The allylic connectivity of 10 was confirmed by the ${ }^{3} J_{C-H^{-}}$ $\mathrm{HMBC}$-coupling between the anomeric mannose $\mathrm{C} 1$ carbon and the allylic hydrogen $\mathrm{C} 5 \mathrm{H}$ of the cyclopentene ring. For $\alpha$-homoallyl-manQ 11, a ${ }^{1} J_{C-H}$-coupling of $170.3 \mathrm{~Hz}$ was observed at the anomeric centre, proving its $\alpha$-configuration. In addition, with $\delta=5.04 \mathrm{ppm}$, the chemical shift of the anomeric proton $\mathrm{C} 1 \mathrm{H}$ of $\mathbf{1 1}$ was as expected higher than the shift observed for the corresponding $\beta$ anomer $3(\delta=4.72 \mathrm{ppm})$. Homoallylic connectivity of 11 was confirmed by observation of an ${ }^{3} J_{C-H^{-}}$ HMBC-coupling of the anomeric carbon $\mathrm{C} 1$ and the homoallylic hydrogen $\mathrm{C} 4 \mathrm{H}$ of the cyclopentene moiety. The structure of $\alpha$-allyl-manQ 4 was proven based on an anomeric ${ }^{1} J_{C-H}$-coupling of $170.0 \mathrm{~Hz}$, indicating its $\alpha$-configuration. Here, the chemical shift of the anomeric proton $\mathrm{C} 1 \mathrm{H}(\delta=4.97 \mathrm{ppm})$ was again higher than that of the corresponding $\beta$-anomer $10(\delta=4.70 \mathrm{ppm})$, thereby also confirming the $\alpha$-configuration of 4 . The allylic connectivity of 4 was confirmed by an ${ }^{3} J_{C-H}-H M B C$ signal resulting from a coupling of the anomeric mannose carbon $\mathrm{C} 1$ with the allylic hydrogen $\mathrm{C} 5 \mathrm{H}$ of the cyclopentene ring.

With all four possible manQ isomers now available ( $\alpha / \beta$-allyl-manQ 4 and 10, and $\alpha / \beta$-homoallyl-manQ 3 and 11), we next performed individual co-injection studies using again a digest of total RNA from mouse liver as reference material, and the UHPLC-MS/MS-method described above for analysis (Fig. 5). While for $\beta$-allyl-manQ 10 and $\alpha$-homoallyl-manQ 11 LC-MS signals were obtained that were well-separated both from galQ 2 and natural manQ (Fig. 5a), we discovered to our delight a full signal overlap of our synthetic $\alpha$-allyl-manQ 4 with the natural manQ compound (Fig. 5b) We finally 
confirmed the overlap of the two compound signals with a second HPLC-MS-method using a different HPLC separation column and gradient (see Supplementary Figure 2). Here, too, the synthetic material co-eluted with the natural manQ compound. These co-injection experiments therefore show that the natural manQ compound present in the anticodon loop of tRNA ${ }^{\text {Asp }}$ has the chemical structure $\mathbf{4}$ (see Fig. $1 \mathbf{d}$ and $\mathbf{4 a}$ ). The configuration of its anomeric centre is in fact $\alpha$, not $\beta$. In addition, the mannosyl moiety of natural manQ is connected to the allylic hydroxyl group of the cyclopentene unit, and not to the homoallylic hydroxyl group as in galQ.
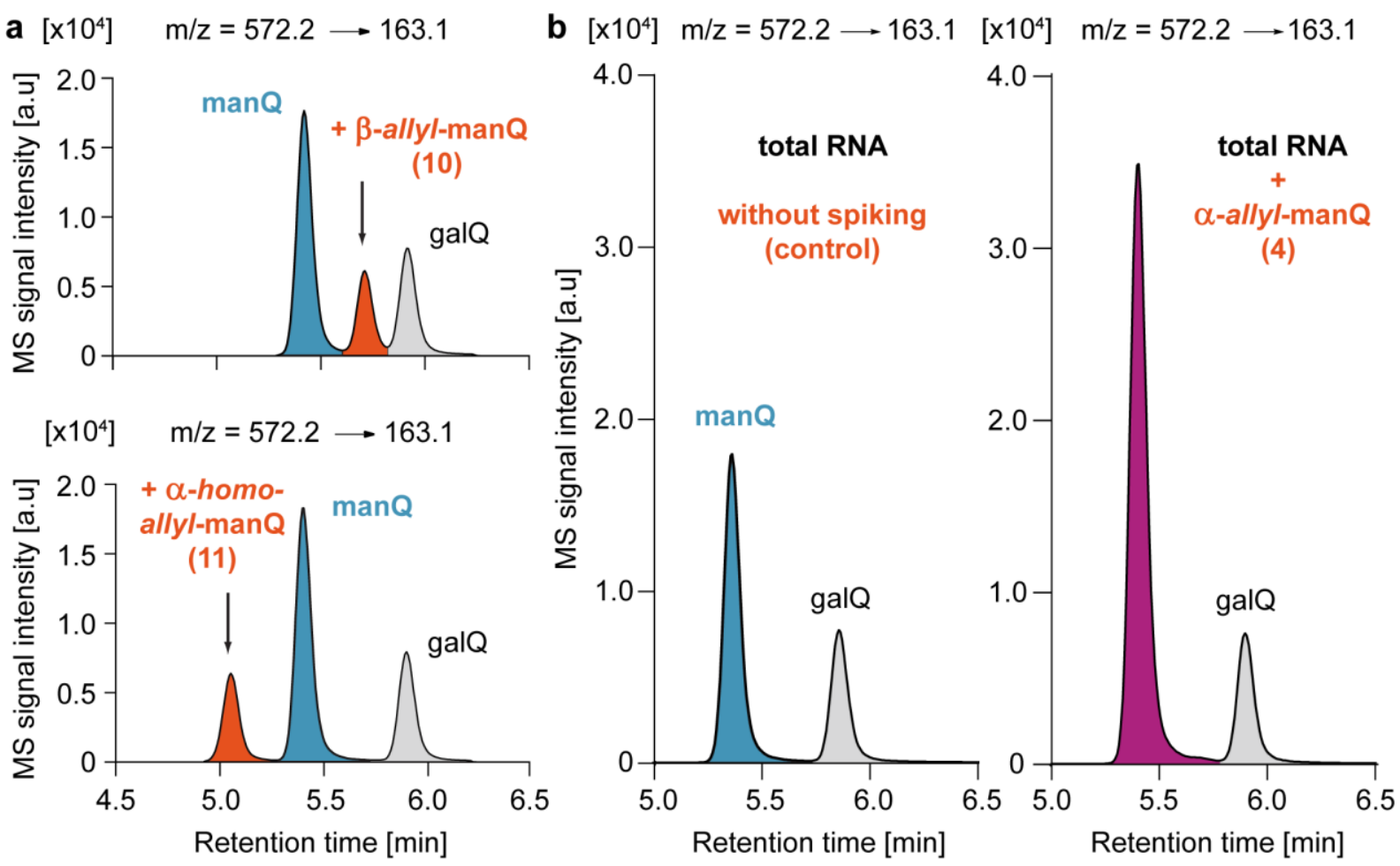

Figure 5. Chromatograms resulting from the co-injection of the synthetic manQ compounds 10, 11, and $\mathbf{4}$ with enzymatically digested total RNA from mouse liver analysed by UHPLC-MS/MS. a, Co-injection of $\beta$-allyl-manQ 10 and $\alpha$-homoallyl-manQ 11 show the appearance of a third peak, these compounds are therefore different from natural manQ; $\mathbf{b}$, Co-injection of $\alpha$-allyl-manQ $\mathbf{4}$ with digested total RNA from mouse liver shows a complete signal overlap of $\mathbf{4}$ and natural manQ, thereby leading to an increased signal intensity of manQ (right) in comparison to the control sample without synthetic standard (left). This result shows that our synthetic compound $\mathbf{4}$ is identical to the naturally occurring manQ nucleoside and establishes its $\alpha$-allyl-connectivity. 


\section{Conclusion}

Based on these experiments, we showed that the widely accepted $\beta$-homoallyl structure reported in literature for the non-canonical RNA nucleoside manQ is not correct. Instead, natural manQ is an $\alpha$-allyl-mannoside (4, Fig. 1d). Its connectivity is therefore maximally different from the $\beta$-homoallylcompound galQ (2, Fig. 1b). The elucidation of the correct manQ structure and the availability of synthetic material will be the basis for illuminating the biological functions of this hypermodified RNA nucleoside.

\section{Methods}

In order to obtain RNA samples for LC-MS-analysis, mice livers were removed and immediately snapfrozen in liquid nitrogen. The subsequent isolation of total RNA, as well as the isolation of total RNA from HEK cells, were performed using commercially available TriReagent from Sigma Aldrich as recently described by us. Enzymatic digestion of the total RNA down to the nucleoside level was achieved with the commercially available Nucleoside Digestion Mix from New England Biolabs. Protocols and additional details for sample preparation can be found in the Supplementary Information. For co-injection experiments, manQ-standards synthesized by us were spiked to digested total RNA samples. Details of this procedure are listed in the Supplementary Information. LC-MSmeasurements were performed on a QQQ-UHPLC-MS/MS system and repeated on an Orbitrap LC-MS system. For additional details on the devices and methods used see Supplementary Information. Synthetic procedures for the preparation of the manQ-compounds 3, 4, 10 and 11 as well as characterization data can be found in the Supplementary Information, together with synthetic procedures and characterization data for all compounds newly synthesized in the course of this study.

\section{Data availability}

All data generated and analysed during this study are included in this article and its Supplementary Information. Chromatograms of co-injection experiments are provided in the article figures and Supplementary Information. Synthesis protocols and NMR-spectra for all relevant compounds, proving their chemical identity and bulk purity, are given in the Supplementary Information. 


\section{References}

$1 \quad$ Crick, F. Central Dogma of Molecular Biology. Nature 227, 561-563, (1970).

2 Cobb, M. 60 years ago, Francis Crick changed the logic of biology. PLoS Biol. 15, e2003243, (2017).

3 Boccaletto, P. et al. MODOMICS: a database of RNA modification pathways. 2017 update. Nucleic Acids Res. 46, D303-D307, (2018).

$4 \quad$ Cantara, W. A. et al. The RNA modification database, RNAMDB: 2011 update. Nucleic Acids Res. 39, D195-D201, (2011).

5 Sarin, L. P. \& Leidel, S. A. Modify or die?--RNA modification defects in metazoans. RNA Biol. 11, 1555-1567, (2014).

6 Rozov, A. et al. Novel base-pairing interactions at the tRNA wobble position crucial for accurate reading of the genetic code. Nat. Commun. 7, 10457, (2016).

7 Agris, P. Wobble position modified nucleosides evolved to select transfer RNA codon recognition: A modified-wobble hypothesis. Biochimie 73, 1345-1349, (1991).

8 Yarian, C. et al. Accurate Translation of the Genetic Code Depends on tRNA Modified Nucleosides. J. Biol. Chem. 277, 16391-16395, (2002).

9 Duechler, M., Leszczyńska, G., Sochacka, E. \& Nawrot, B. Nucleoside modifications in the regulation of gene expression: focus on tRNA. Cell. Mol. Life Sci. 73, 3075-3095, (2016).

Thumbs, P. et al. Synthesis of galactosyl-queuosine and distribution of hypermodified Qnucleosides in mouse tissues. Angew. Chem. Int. Ed. , (2020).

Klepper, F., Jahn, E. M., Hickmann, V. \& Carell, T. Synthesis of the transfer-RNA nucleoside queuosine by using a chiral allyl azide intermediate. Angew. Chem. Int. Ed. 46, 2325-2327, (2007).

12 Nishimura, S. Structure, Biosynthesis, and Function of Queuosine in Transfer RNA. Prog. Nucleic Acid Res. Mol. Biol. 28, 49-73, (1983).

13 Harada, F. \& Nishimura, S. Possible anticodon sequences of tRNAHis, tRNAAsn, and tRNAAsp from Escherichia coli. Universal presence of nucleoside $O$ in the first position of the anticodons of these transfer ribonucleic acid. Biochemistry 11, 301-308, (1972).

14 Katze, J. R., Basile, B. \& McCloskey, J. A. Queuine, a modified base incorporated posttranscriptionally into eukaryotic transfer RNA: wide distribution in nature. Science 216, 55-56, (1982).

Salinas-Giegé, T., Giegé, R. \& Giegé, P. tRNA biology in mitochondria. Int. J. Mol. Sci. 16, 45184559, (2015).

16 Kasai, H. et al. Structure of the modified nucleoside $Q$ isolated from Escherichia coli transfer ribonucleic acid. 7-(4,5-cis-Dihydroxy-1-cyclopenten-3-ylaminomethyl)-7-deazaguanosine. Biochemistry 14, 4198-4208, (1975).

17 Morris, R. C., Brown, K. G. \& Elliott, M. S. The effect of queuosine on tRNA structure and function. J. Biomol. Struct. Dyn. 16, 757-774, (1999).

18 Meier, F., Suter, B., Grosjean, H., Keith, G. \& Kubli, E. Queuosine modification of the wobble base in tRNAHis influences 'in vivo' decoding properties. EMBO J. 4, 823-827, (1985).

19 Tuorto, F. et al. Queuosine-modified tRNAs confer nutritional control of protein translation. EMBO J. 37, e99777, (2018).

20 Zaborske, J. M. et al. A Nutrient-Driven tRNA Modification Alters Translational Fidelity and Genome-wide Protein Coding across an Animal Genus. PLoS Biol. 12, e1002015, (2014).

21 Marks, T. \& Farkas, W. R. Effects of a diet deficient in tyrosine and queuine on germfree mice. Biochem. Biophys. Res. Commun. 230, 233-237, (1997).

22 Rakovich, T. et al. Queuosine deficiency in eukaryotes compromises tyrosine production through increased tetrahydrobiopterin oxidation. J. Biol. Chem. 286, 19354-19363, (2011).

23 Farkas, W. R. Effect of diet on the queuosine family of tRNAs of germ-free mice. J. Biol. Chem. 255, 6832-6835, (1980).

24 Fergus, C., Barnes, D., Alqasem, M. \& Kelly, V. The Queuine Micronutrient: Charting a Course from Microbe to Man. Nutrients 7, 2897-2929, (2015). 
25 Reyniers, J. P., Pleasants, J. R., Wostmann, B. S., Katze, J. R. \& Farkas, W. R. Administration of exogenous queuine is essential for the biosynthesis of the queuosine-containing transfer RNAs in the mouse. J. Biol. Chem. 256, 11591-11594, (1981).

26 Tuorto, F. \& Lyko, F. Genome recoding by tRNA modifications. Open Biol. 6, (2016).

27 Barbieri, I. \& Kouzarides, T. Role of RNA modifications in cancer. Nat. Rev. Cancer 20, 303-322, (2020).

28 Suzuki, T. The expanding world of tRNA modifications and their disease relevance. Nat. Rev. Mol. Cell Biol. 22, 375-392, (2021).

29 Kasai, $\mathrm{H}$. et al. Letter: The structure of $\mathrm{Q}^{*}$ nucleoside isolated from rabbit liver transfer ribonucleic acid. J. Am. Chem. Soc. 98, 5044-5046, (1976).

30 Okada, N., Shindo-Okada, N. \& Nishimura, S. Isolation of mammalian tRNAAsp and tRNATyr by lectin-Sepharose affinity column chromatography. Nucleic Acids Res. 4, 415-423, (1977).

31 Crich, D. \& Sun, S. Direct Formation of $\beta$-Mannopyranosides and Other Hindered Glycosides from Thioglycosides. J. Am. Chem. Soc. 120, 435-436, (1998).

32 D. Crich. M. de la Mora, R. C. Synthesis of the mannosyl erythritol lipid MEL A; confirmation of the configuration of the meso-erythritol moiety. Tetrahedron 58, 35-44, (2002).

33 Tvaroska, I. \& Taravel, F. R. Carbon-Proton Coupling Constants In The Conformational Analysis of Sugar Molecules. Adv. Carbohydr. Chem. Biochem. 51, 15-61, (1995).

34 Okada, N. \& Nishimura, S. Enzymatic synthesis of $Q$ nucleoside containing mannose in the anticodon of tRNA: isolation of a novel mannosyltransferase from a cell-free extract of rat liver. Nucleic Acids Res. 4, 2931-2938, (1977).

\section{Acknowledgements}

This project has received funding from the European Research Council (ERC) under the European Union's Horizon 2020 research and innovation program under grant agreement EpiR, No 741912. Further financial support was obtained from the Deutsche Forschungsgemeinschaft (DFG, German Research Foundation) via SFB1309 (Project-ID 325871075) and SPP 1784 (Project-ID 277203618). We thank the Volkswagen Foundation for generous support through the EvoRib VW-project.

\section{Author contributions}

M.H. and P.T. synthesized the compounds. E.K. and M.W. developed UHPLC-MS/MS methods for detection of the manQ derivatives. M.W., E.K., and M.H. performed the LC-MS co-injectionexperiments. T.E. performed the metabolic feeding experiments and did the isolation of total RNA from mouse liver and HEK cells. M.W. developed HPLC-MS methods and performed the LC-MS analysis of the feeding experiments. T.C. and M.M. supervised the experiments and the project. T.C. designed the project. T.C. wrote the paper with contributions from M.H., M.W., T.E., E.K., and M.M..

\section{Competing Interests}

The authors declare no competing interests. 